Sādhanā Vol. 39, Part 1, February 2014, pp. 27-37. (C) Indian Academy of Sciences

\title{
Providing an efficient intelligent transportation system through detection, tracking and recognition of the region of interest in traffic signs by using non-linear SVM classifier in line with histogram oriented gradient and Kalman filter approach
}

\author{
A H MAZINAN* and M SARIKHANI \\ Islamic Azad University (IAU), South Tehran Branch, Tehran, Iran \\ e-mail: mazinan@azad.ac.ir
}

MS received 27 January 2013; revised 17 August 2013; accepted 21 August 2013

\begin{abstract}
With a focus on new researches in the area of intelligent transportation systems (ITS), an efficient approach has been investigated here. Based on the present view point, analysis of traffic signs are first considered via intelligence based approach, which is carried out through three main stages including detection, tracking and recognition, respectively, in this research. The key role of detection is to identify traffic signs by classification of road sign shapes in accordance with their signatures. This classification consists of four different shapes of circle, semicircle, triangle and square, as well. The linear classification of traffic sign is also carried out via support vector machine (SVM) by using one against all (OAA), since the present SVMs classifiers realized via linear kernel. The next step is to track traffic sign. It should be noted that this technique is now developed to reduce the searching mode in case of the whole area to be optimized its computational processing, consequently. This research work is investigated by realizing Kalman filter approach, where, finally, in recognition step, a feature of the region of interest (ROI) has been extracted for SVM classification. Histogram of oriented gradient (HOG) is realized in organizing the approach, as long as Gaussian kernel is also developed for non-linear SVM classifier.
\end{abstract}

Keywords. RGBN; signature; bounding box; SVM; ROI; HOG.

\section{Introduction}

In car industry, some efforts such as safety, travel time, traffic congestion and fuel consumption have been made to be optimized. In the last 15 years, a number of cars are moving to Intelligent Transportation System (ITS). Among the activities, detection, recognition and tracking of

*For correspondence 
traffic signs are so important to investigate. In this system, colour is an excellent tool for detection system. However, another activity such as edge detection has been carried out. In fact, in the first time, as suggested in Zakir (2011), Ruta et al (2008), Ghica et al (1995) and Fang et al (2003) by using the threshold of colour RGB and HSI spaces, segmentation of the image is carried out, but it had many errors because of the extreme sensitivity of the colour space to illumination changes. After applying threshold of the colours, the second step involves computing the connected components and after a certain limitation, such as the size or aspect ratio, it is entered to stage detection geometric shapes for classification. There are several techniques used for classification of traffic signs such as back propagation or winner-takes-all sub-network (Hopfield network) Kellmeyer \& Zwahlen (1994) and Ghica et al (1995) in neural networks. In this paper, by using signature (Gil-Jiménez et al 2005, 2011), shapes are introduced to Support Vector Machine (SVM) system (Maldonado Bascón et al 2007). In the next stage, after shape detection, a total of image successive is not searched in the next frame and only special area is detected, since their signs are existed, applying Kalman filter for tracking targets. In recognition as stated (Hou 2009), it is used by feature extraction such as Scale-Invariant Feature Transform (SIFT) for classification. In this research, Histogram Oriented Gradients (HOG) that is used for pedestrian detection in the first time has been realized (Dalal \& Triggs 2005). Consequently our system consists of three stages (see figure 1).

\subsection{Detection}

This stage consists of separated road signs from their background. This process can be carried out using three different features: shape, colour and both shape and colour information. Here, colour information has been only used in segmentation, once detection stage has the following problems:

(a) Rotation out-of-plane: this occurs when camera optical axis is not perpendicular to plan of road sign.

(b) Occlusions: part of the road sign is lost through objects such as car, or tree and so on, thus detection and recognition become too difficult.

(c) Variable lighting conditions: the colour of the road sign changed to different levels of incident light.

(d) Vandalism and aging: the information of the road sign is misrepresented, thus the classification process may be impossible or incorrect. These problems are shown in figure 2.

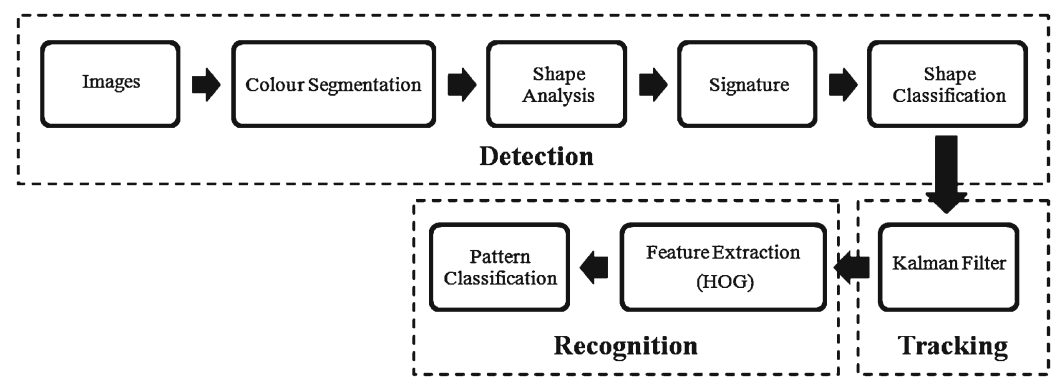

Figure 1. Block diagram of system. 


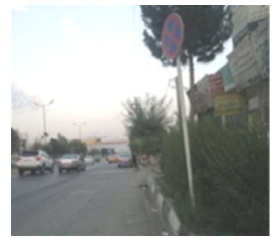

(a)

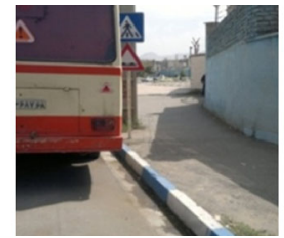

(b)

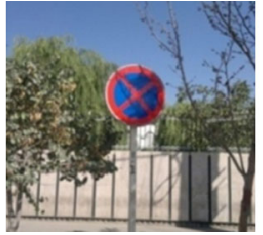

(c)

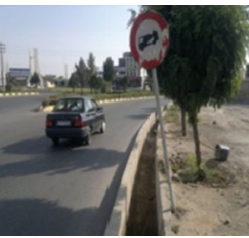

(d)

Figure 2. Detection problems. (a) Rotation out-of-plane, (b) occlusions, (c) illumination problems light, (d) vandalism.

\subsection{Colour segmentation}

The main task in traffic sign detection consists of segmentation of the traffic sign in environment. Because the use of colour analysis is basic, this approach is applied to many works in road sign detection (Gómez Moreno \& Maldonado Bascón 2010). As mentioned in the section, different colour spaces can be employed for segmenting traffic signs such as RGB, HSI and YUV. Here, for enhancing the desired colour in an image, the threshold of normalized RGB (RGBN) colour space has been applied. By using the histogram for the three primary colours of yellow, red and blue, the threshold value is determined. Threshold value of RGBN is given in equations (1)(3). Also, as illustrated in figure 3, the results are viewable. In this system, variable lighting conditions have been reduced by this colour space, i.e.,

$$
\begin{aligned}
& \operatorname{Red}(i, j)=\left\{\begin{array}{lc}
\text { True, } & \text { if } r(i, j) \geq 0.41 \\
& \text { and } g(i, j) \leq 0.32 \\
\text { False, } & \text { otherwise }
\end{array}\right. \\
& \text { Blue }(i, j)=\left\{\begin{array}{l}
\text { True, } \text { if } H(i, j) \geq 0.42 \\
\text { False, } \text { otherwise }
\end{array}\right. \\
& \text { Yellow }(i, j)=\left\{\begin{array}{l}
\text { True, } \text { if }(r(i, j)+g(i, j)) \geq 0.8 \\
\text { False, } \text { otherwise }
\end{array} .\right.
\end{aligned}
$$

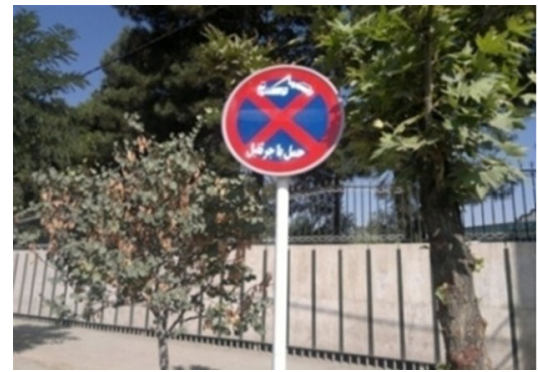

(a)

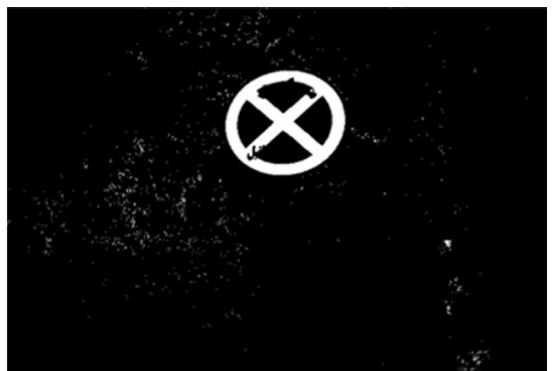

(b)

Figure 3. Colour thresholding. (a) Original image, (b) colour segmentation. 


\subsection{Shape detection}

The goal of the shape detection stage is the identification of the geometric shape of all blobs obtained on the segmentation system. This work is carried out to increase the accuracy recognition classification of traffic sign, data base in the proposed case which are for the Iranian traffic sign set, since these shapes are typically taken as the equilateral square, triangle and circle, as well. Besides, semicircle is added, once some signs such as 'end of prohibition' are divided to two semicircles in segmentation step. After segmentation each labelled region is a possible candidate for a traffic sign but the number of candidates can be reduced by eliminating regions so large and so small, while a sign could not be identified out of them. In this research work, a threshold of $\max =5700$ pixels and $\min =200$ pixels are taken. The number of candidates can be reduced even more. All regions whose aspect ratio (height/wide) is out of 0.8 and 1.2 are discarded with the traffic sign sets. In the next step, the signature of shape classification is introduced.

\subsection{Blob signature}

Signature is a 1-D function to represent the boundary of the shape and may be generated in various ways. For example, to plot distance from the centroid to the boundary as a function of angle, the results are shown in figure 4. Regardless of how a signature is generated, the basic idea is to reduce the boundary representation to a 1-D function. The structure of figure 4 consists of 50 samples that angle of per two samples is 7.2 degree. If this angle could be increased, its accuracy will be poor. Signatures generated by approach describing here are invariant to translation, but they are depended on scaling and rotation (Gonzalez \& Woods 2008). Since for rotation invariant by equation (4) in digital signal processing, the absolute value of FFT (AbFFT) is used. Because the signature is a real signal, the (AbFFT) is symmetric, so the half of the samples is acquired. Also for scaling invariant, it should normalize (25 samples). Here normalization is carried out with total energy for each signature. Detection stages are shown in figure 1.

\subsection{Shape classification}

Shape classification is carried out, since recognition system could be stricter. Final goal in this stage is the classification of the normalized AbFFT of the signature to the classes where there is four classes including square, circle, semicircle and finally triangle. Here, in the classification, SVM is realized. The present SVM is the pattern classification techniques based on mathematical foundations of statistical learning theory. The SVM learns a separating hyperplane to maximize the margin and to produce good generalization ability. Due to the good
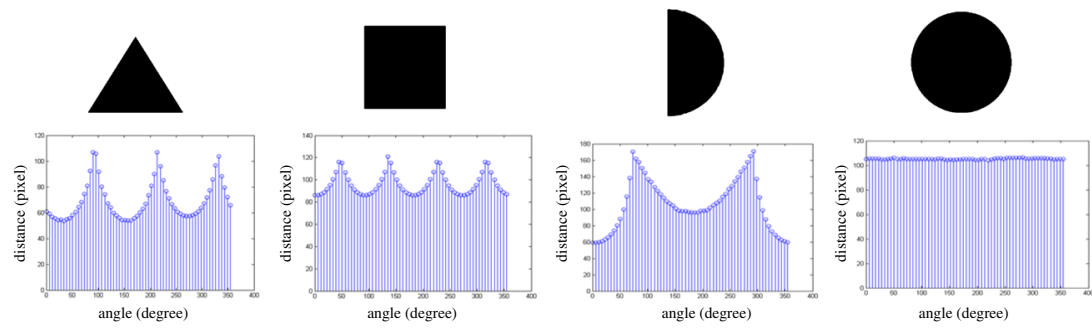

Figure 4. Reference shapes and their signatures. 


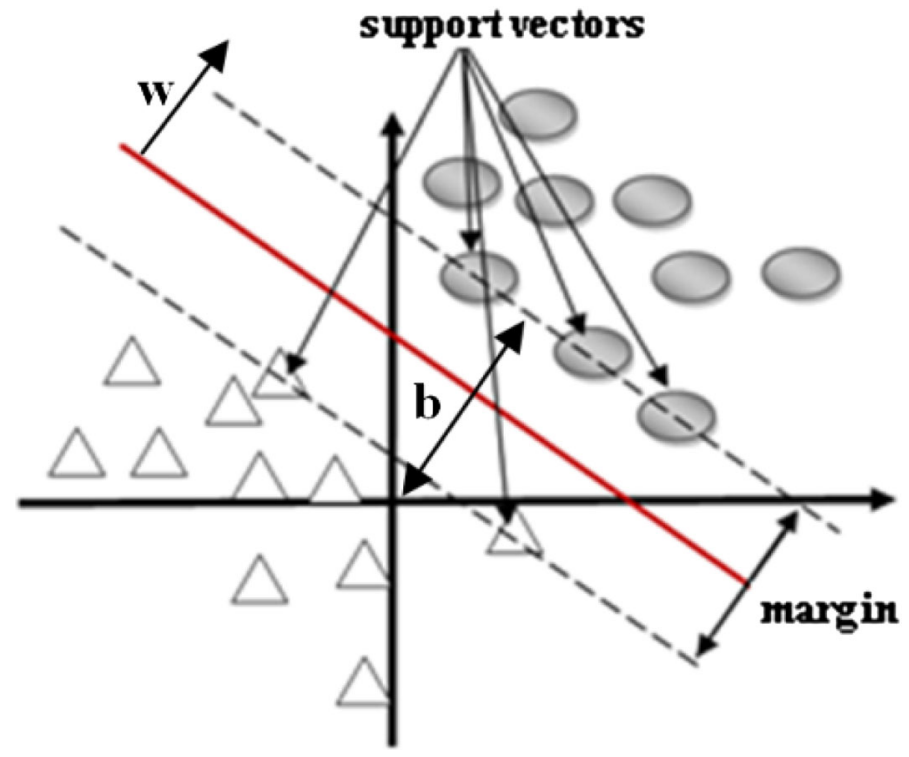

Figure 5. Binary classification for SVM.

generalization performance on a large number of real-life data and also due to the fact that the approach is properly motivated, it has been used for a wide range of applications. This theory was first proposed by Vapnik (2000). Although SVMs are strictly explained in Wang et al (2005) here its main concepts are given to be clear in the present research. The training data is given as a data set $S$ of points $\mathbf{x}_{i} \in\{\mathbf{R}\}^{d}$ ( $d$ is the dimension of the problem) with the label $\mathbf{y}_{i} \in\{-1,+1\}$ in a binary classification, where $i=1, \ldots, l$ ( $l$ is the number of training vector). Here two class positive $(+1)$ and negative $(-1)$ are taken, (see figure 5). Distance from the nearest of negative plane and positive plane is called margin, and the data points are closest to the margins plane which are support vectors (SV). The goal is to maximize the margin hyperplane and to find a classification function based on the present $\mathrm{SV}$, i.e.,

$$
\begin{aligned}
& y[n] \rightarrow Y(\omega) \\
& y[n-n 0] \rightarrow Y(\omega) e^{j \omega n 0 \stackrel{\text { Absolute }}{\longrightarrow}} Y(\omega) .
\end{aligned}
$$

Decision function is given by:

$$
f(x)=\operatorname{sgn}\left(\sum_{i=1}^{l} \alpha i y i K(x i, x)+b\right),
$$

where $x i$ and $y i$ are the SV, $l$ is the number of support vectors, $b$ is called bias, $x$ is the vector to be classified and the function $K($.$) is the kernel, which its non-linear decision surface can be$ obtained. The optimal separating hyperplane $\langle w, x i\rangle+b=0$ is given by solving the following optimization problem:

$$
\begin{array}{ll}
\min _{w, b, \zeta} & \frac{1}{2}\|w\|^{2}+C \sum_{i=1}^{l} \zeta_{i} \\
\text { Subject to } & y i(\langle w, x i\rangle+b) \geq 1-\zeta_{i} \\
& \zeta_{i} \geq 0, \quad i=1, \ldots, l
\end{array}
$$




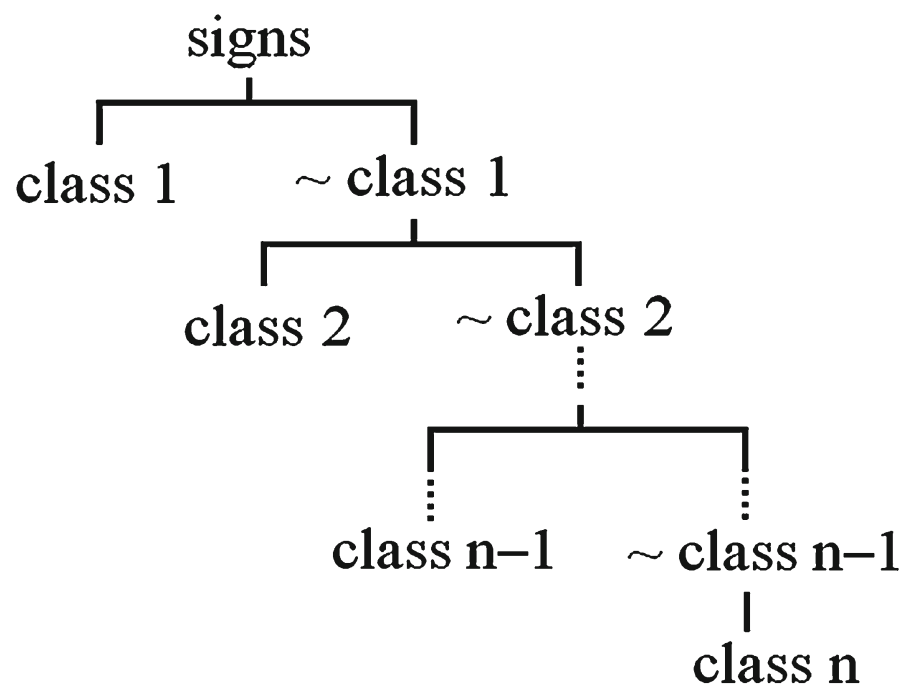

Figure 6. Structure of OAA.

where, $\zeta_{i}$ is a slack variables and $C$ is a penalty parameter. At first, the optimization problem (6) is converted into dual form by introducing Lagrange multipliers and then the dual optimal problem is solved by quadratic programming $(\mathrm{QP})$ methods. The dual problem is expressed as:

$$
\max (\alpha)=\sum_{i=1}^{l} \alpha_{i}-\frac{1}{2} \sum_{i}^{l} \sum_{j}^{l} \alpha_{i} y_{i} \alpha_{j} y_{j}\left\langle x_{i}, x_{j}\right\rangle
$$

Subject to

$$
\alpha_{i} \in[0, C], \quad i=1, \ldots, l .
$$

Because number of road sign shapes is more than two classes, multi-class classifier is used such as 'one against all' (OAA), 'one against one' (OAO). In this research, it is used OAA that it is shown in figure 6. For training data of shape classification a set composed of: 120 triangles, 120 circles, 120 semicircles and 120 squares are taken with different deformations.

\section{Tracking}

To provide accurate localization and fast computation of detection process, the approach of Kalman filtering is used to predict road sign by camera information. By applying this method, the detection process can narrow down the search space for each image. The Kalman filter is involved to two phases: time update and measurement update. The time update procedure is based on the dynamic equation which is derived from the spatial constraints from the two successive frames. The measurement update is based on the image processing location in the proximity of the predicted candidate region. By computing the geometric relationship of the road sign 
between adjacent frames, the framework finds the candidate region for the road sign in the next frame. A camera is mounted on the vehicle and its optical axis is calibrated to be parallel to the horizontal plane of the vehicle. Equations (8) and (9) are for time updating (predict) and equations (10)-(12) are for measurement updating (correction). The five equations for Kalman filter are (Wang et al 2005):

$$
\begin{gathered}
\hat{x}_{\bar{k}}=A K_{\hat{k}-1}+B u_{k-1} \\
P_{\bar{k}}=A P_{k-1} A^{T}+Q,
\end{gathered}
$$

whereas $\hat{x}_{\bar{k}}$ is a priori state estimate at step $k$ with given knowledge from step $k-1$ in (8) and $\hat{x}_{k}$ is a posteriori state estimate at step $k$ given measurement $z_{k}$.

$$
\begin{gathered}
K_{k}=P_{\bar{k}} H_{k}^{t}\left(H_{k} P_{\bar{k}} H_{k}^{t}+R_{k}\right)^{-1} \\
X_{\hat{k}}=\hat{X}_{\bar{k}}+K_{k}\left(Z_{\bar{k}} H_{\hat{x} \bar{k}}\right) \\
P_{k}=\left(I-k_{k} H_{k}\right) P_{\bar{k}},
\end{gathered}
$$

whereas $\left(z_{\bar{k}} H_{\hat{x} \bar{k}}\right)$ is measurement innovation or residual that reflects the discrepancy between the predicted measurement $H_{\hat{x} \bar{k}}$ and the actual measurement $z_{k} . P_{k}$ is a posteriori estimate error covariance and $P_{\bar{k}}$ is a priori estimate error covariance. $K_{k}$ is Kalman gain which is calculated by minimizing the a posteriori error covariance. The parameters which are introduced here are all designed as: the properties of the road sign in the successive frames at each time instance (frame), as it can be characterized by its position and size. Let $\left(x_{t}, y_{t}, h_{t}, w_{t}\right)$ represent the road sign pixels position (it's centroid) and size at time $t$. The state vector at time $t$ can therefore be represented as $x=\left(x_{t}, y_{t}, h_{t}, w_{t}\right)^{T}$. The system can therefore be modelled as:

$$
x_{t}=A x_{t-1}+\omega_{t}
$$

where $\omega_{t}$ represents system perturbation which comes from the drive direction variance, Distance Measurement Instrument (DMI) measurement error, etc. It is further assumed that the image processing estimates:

$$
z_{t}=H x_{t}+v_{t}
$$

Here, $v_{t}$ represents measurement uncertainty. Specifically, the position of the current frame $t$ is estimated based on an image processing in the neighbourhood of the predicted position (based on the system model). In this manner, the state vector $x_{t}$, along with its covariance matrix $P_{t}$, can be updated using the system model (for prediction) and measurement model (for updating) (figures 7 and 8). 


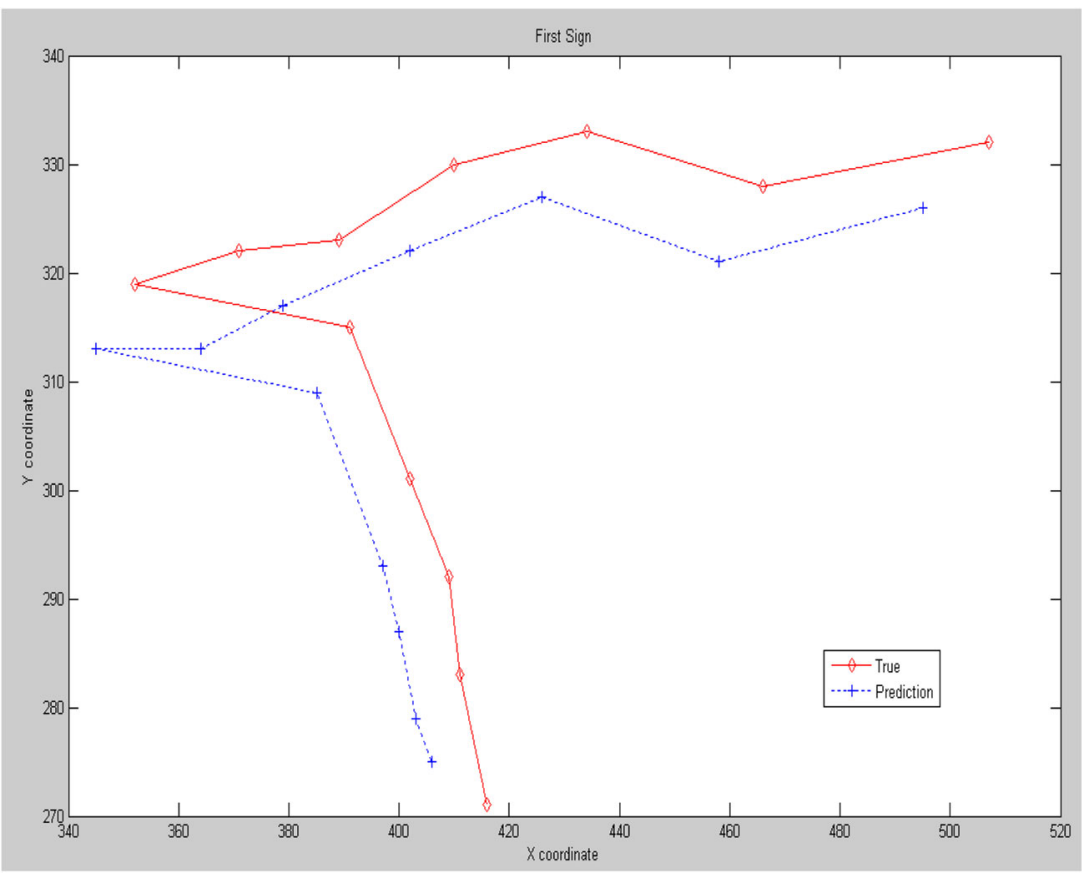

Figure 7. Position prediction for a road sign.

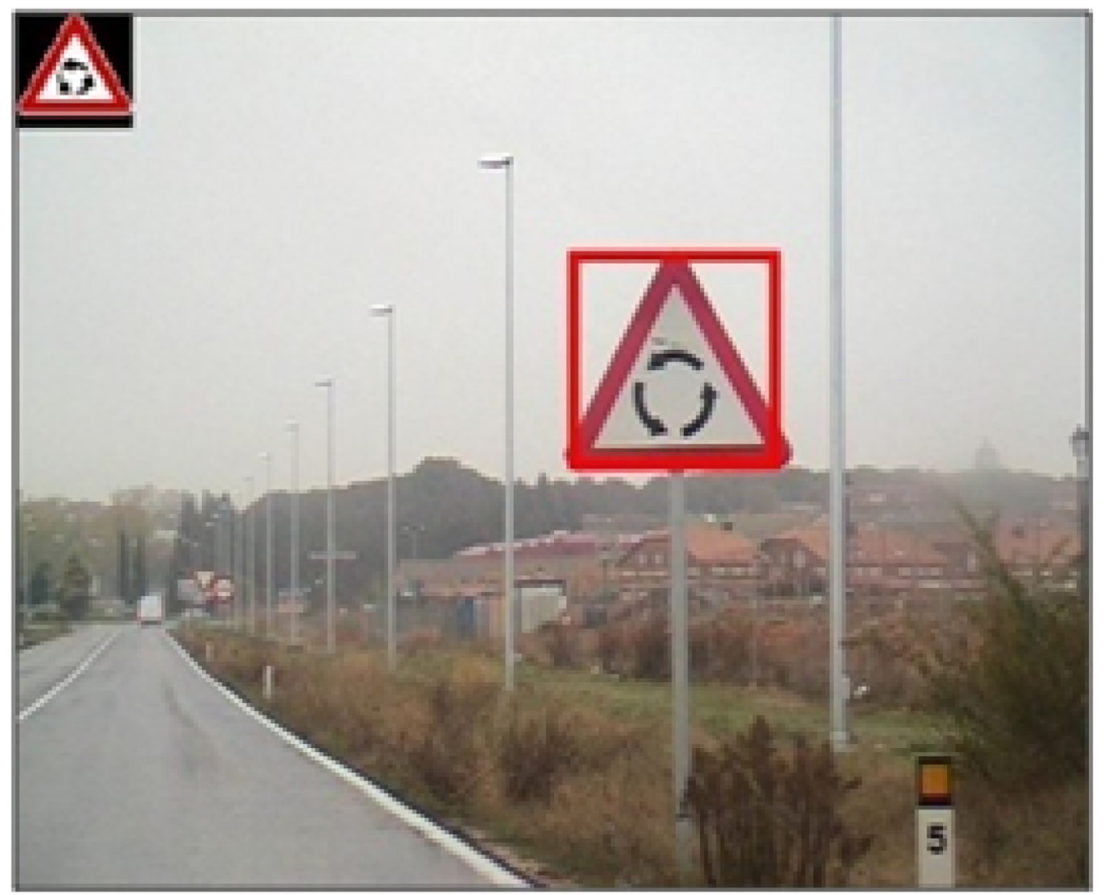

Figure 8. Experimental results in recognition and tracking. 


\section{Recognition}

Once region of interest (ROI) is detected, the next step is preparation of feature extraction for SVM classification. Here, HOG is used which is introduced by Dalal and Triggs for pedestrian detection at first (Dalal \& Triggs 2005). The HOG computation steps are given by: (i) extraction of ROI (the road sign) where this is a RGB image, (ii) gradient and gradient angle computation, (iii) cell splitting (this is implemented by dividing the image window into small spatial regions (cells)), (iv) histogram normalization descriptor block 1, block 2 . . block $n$ (block is divided by the number of cells that these ones can overlap together when final descriptor is written). These stages are illustrated for 'No pedestrians' sign in figure 9. Here, the dimensions of ROI is taken as $50 \times 50$ pixels in RGB image, where a 800 value descriptor with 8-bin histogram for all our ROI is used. Through noise in the some images, training data for SVM classification per class varies between 10 and 15, but some of them define the decision hyperplane as support vectors only. Figure 10 shows training instances for 'Roundabout' signs in the SVM.

Now we train SVM with final descriptor of the computed HOG of ROI. Also we used against all SVMs classifier. Because here accuracy of a Gaussian kernel is more than of the linear kernel, we have used of Gaussian kernel. This kernel is defined as follows:

$$
K(x, y)=e^{\frac{-\|x-y\|^{2}}{2 \sigma^{2}}} .
$$

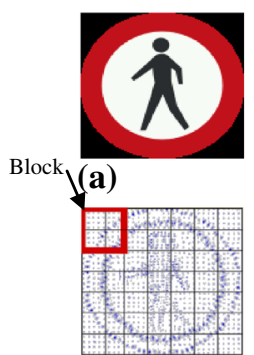

(d)

Final descriptor: $[0.10 .5 \ldots .8]$

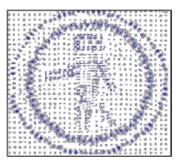

(b)

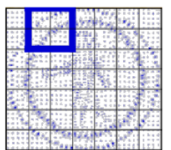

(e)

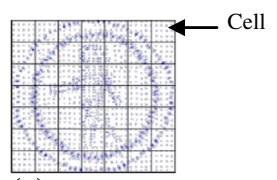

(c)

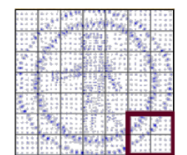

(f)

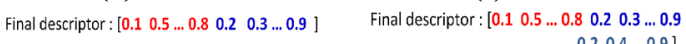

Figure 9. Stages of HOG descriptor. (a) original image, (b) gradient angle computation, (c) cell splitting, (d) block 1, (e) block 2, (f) block $n$.
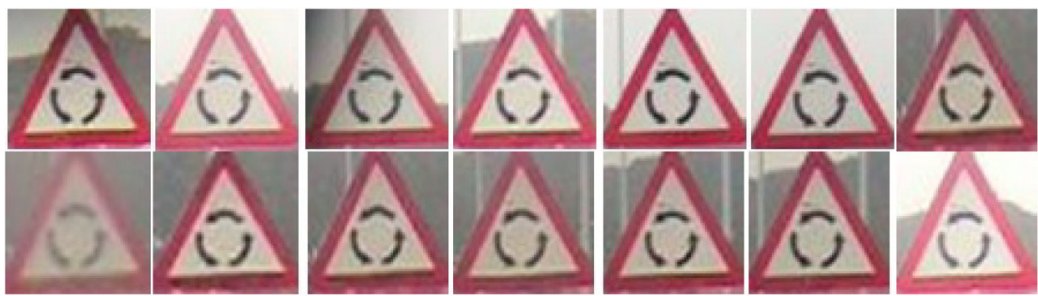

Figure 10. Training data of warning signs 'Roundabout' for support vector machine. 
Table 1. Results for segmentation.

\begin{tabular}{lccc}
\hline Number of video sequence & 1 & 2 & 3 \\
\hline Number of signs & 10 & 40 & 40 \\
RGBN & $80 \%$ & $87 \%$ & $96 \%$ \\
HSI & $79 \%$ & $82 \%$ & $94 \%$ \\
\hline
\end{tabular}

Table 2. The accuracy for detection and recognition for total system.

\begin{tabular}{lcc}
\hline & Sunny & Night \\
\hline Number of traffic signs & 50 & 40 \\
Recognitions of traffic signs & 48 & 35 \\
\hline
\end{tabular}

\section{Results}

The experimental results have been obtained using an implementation based on MATLAB language and the SVM library (Rakotomamonjy 2009). We achieve a mean processing time of 1.9 s per frame on a $1.6 \mathrm{GHz}$ T2050 and $1 \mathrm{~GB}$ of RAM, where the frame dimensions are $720 \times 576$ pixels. Several video sequence are in Ljung (2007) for testing system. This system, which involves a mixture of computer vision and pattern recognition problems, was able to extract road signs from still images of complex scenes subject to uncontrollable illumination. The normalized RGB and HSI space in colour segmentation is used and the RGBN is taken more accurate than HSI. Table 1 summarizes this result. Here, the segmentation system is carried out for different weather condition, since video sequence 1 is a fog day, video sequence 2 is captured at night and finally video sequence 3 corresponds to a sunny lighting day. Also computation time in RGBN was faster than HSI (about $0.6 \mathrm{~s}$ ).

In this case, the linear kernel is realized in the shape classification through 'One Against All' classifier under $C=1000$. Also, to ensure the well conditioning of the linear system (with bound constraints on $\alpha_{i}$ ), it is induced by the QP problem and ridge regularization, since parameter $\lambda$ is used. Typical value of $\lambda$ is $10^{-7}$. Here, 480 training shapes for 4-class are tested. For the training process of SVMs, the result of Rakotomamonjy (2009) and Maldonado Bascón et al (2007) is used. For the classification of shape with binary images, the average performances of $98 \%$ accuracy are acquired for test sets.

In this paper, the SVM classification is realized with a Gaussian kernel for recognition system, whit $C=1000, \lambda=10^{-7}$ and $\sigma=8$ (for Gaussian kernel). HOG feature extraction has 800 samples where ROI is resized to images with $50 \times 50$ pixels. Finally, in table 2 , it is shown that the accuracy rate and computation time per frame for detection, tracking and recognition in total system. In this experiment, a video sequence is captured in sunny conditions and dark conditions.

\section{Conclusion}

In this research, traffic sign detection and recognition system which can help in creating a road sign inventory was developed, implemented and evaluated. In the colour segmentation part, algorithms were developed to segment the image by using RGBN colour spaces and in the shape 
detection part, signature was used to recognize the sign shapes. In the feature extraction for ROI recognizer, HOG was invoked to introduce to the SVM classification. In the classification part, two SVM classifiers were realized, the first stage classifies the sign shape and the second stage classifies the pictogram of the sign. The performance achieved by this classifier, which is about $96 \%$, suites this kind of application. The proposed system opens new frontiers for novel further research in the future. Automation of the recognition of traffic sign is becoming a necessity of road authorities and such a system will be in use in the very near future.

\section{Acknowledgements}

Authors would like to thank the Islamic Azad University (IAU), South Tehran Branch for support. This work is carried out under contract with the Research Department of IAU, South Tehran Branch.

\section{References}

Dalal N and Triggs B 2005 Histograms of oriented gradients for human detection. In: Comp. Vis. and Patt. Recog., C Schmid, S Soatto, C Tomasi (eds) vol. 2, pp. 886-893, INRIA Rhône-Alpes, ZIRST-655, av. de l'Europe, Montbonnot-38334

Fang C, Fuh C, Chen S and Yen P 2003 A road sign recognition system based on dynamic visual model, IEEE Computer Society Conf. Computer Vision and Pattern Recognition, Madison, Wisconsin

Ghica D, Lu S and Yuan X 1995 Recognition of traffic signs by artificial neural network, IEEE Inter. Conf. Neural Networks, Perth, W.A.

Gil-Jiménez P, Lafuente-Arroyo S, Maldonado-Bascón S and Gómez-Moreno H 2005 Shape classification algorithm using support vector machines for traffic sign recognition. Comput. Int. Bioinspired Syst. Lect. Note Comput. Sci. 3512: 873-880

Gil-Jiménez P, Gómez-Moreno H, Rodríguez J A, López-Sastre R J and Bascón S 2011 Evaluation of shape classification techniques based on the signature of the blob. ELSEVIER Signal Processing, 7 June

Gonzalez R C and Woods R E 2008 Digital image processing, Second Edition. Prentice Hall

Gómez Moreno H and Maldonado Bascón S 2010 Goal evaluation of segmentation algorithms for traffic sign recognition. IEEE Trans. Intell. Syst. 11(4): 917-930

Hou Z 2009 An automated road sign inventory system based on computer vision, Thesis

Kellmeyer D and Zwahlen H 1994 Detection of highway warning signs in natural video images using color image processing and neural networks, IEEE World Congress on Computational Intelligence, Orlando, Florida, USA

Ljung D 2007 Road sign images, Available: http://agamenon.tsc.uah.es

Maldonado Bascón S, Lafuente Arroyo S, Jiménez P G, Gómez Moreno H and López Ferreras F 2007 Road-sign detection and recognition based on support vector machines. IEEE Trans. Intell. Trans. Sys. 8(2): 264-278

Rakotomamonjy A 2009 A library for support vector machines, Available: http://asi.insa-rouen.fr/\% 7Earakotom/toolbox/index

Ruta A, Yongmin L and Xiaohui L 2008 Detection, tracking and recognition of traffic signs from video input. Proc. IEEE Conf. ITS, Beijing

Vapnik V 2000 The nature of statistical learning theory. New York: Springer-Verlag

Wang K, Hou Z and Gong W 2005 Automated detection, tracking, and recognition of roadway signs, First Annual Inter-university Symposium on Infrastructure Management (AISIM) University of Waterloo, Waterloo. ON, Canada, 6 August

Zakir U 2011 Automated road sign detection and recognition, $\mathrm{PhD}$ thesis, Loughborough University 